\title{
Why Projects Often Fail Even With High Cost-Contingencies
}

\author{
Edouard Kujawski \\ Systems Engineering Department \\ Lawrence Berkeley National Laboratory
}

\begin{abstract}
In this note we assume that the individual risks have been adequately quantified and the total project cost contingency adequately computed to ensure an agreed-to probability or confidence level that the total project cost estimate will not be exceeded. But even projects that implement such a process are likely to result in significant cost overruns and/or project failure if the project manager allocates the contingencies to the individual subsystems. The intuitive and mathematically valid solution is to maintain a project-wide contingency and to distribute it to the individual risks on an as-needed basis. Such an approach ensures cost-efficient risk management, and projects that implement it are more likely to succeed and to cost less. We illustrate these ideas using a simplified project with two independent risks. The formulation can readily be extended to multiple risks.
\end{abstract}

\section{INTRODUCTION}

Technology projects typically carry multiple residual risks because practical risk-response actions seldom have a $100 \%$ probability of meeting performance, cost, and schedule. Each residual risk has a range of possible outcomes with associated probabilities. Algebraically summing the worst case residual cost risks leads to an excessive total project cost contingency [U.S. Department of Energy, 2000: Practices 8 and 12]. The mathematically correct total project cost-risk is the probabilistic sum of all the residual risks and it is routinely computed using Monte Carlo simulation. The resulting cumulative probability distribution provides the data to determine the total project contingency required to ensure an agreed-to (with customer or funding agency) probability or confidence level that the total project cost estimate will not be exceeded.

Some projects allocate the total project cost contingency to the individual subsystems [Kindinger, 1999]. But once cost contingencies are distributed at the subsystem-level a modified Parkinson's Principle, "money allocated is money spent", is activated. This negates the premise that contingency usage is probabilistic, and the serious consequence is that the cost-risk is not resolved as anticipated. The common sense and mathematically valid solution for efficient costrisk management is to maintain a project-wide contingency and to distribute it to the individual risks on an as-needed basis. Such a solution also incorporates a basic premise of modern portfolio theory [Markowitz, 1959] that can be generalized to projects as advice that less attention should be given to the individual risks (substituted for stocks) and more to the project (substituted for portfolio) as a whole. Critical Chain Project Management [Goldratt, 1997] formalizes analogous concepts and their implementation for project schedule planning.

In this note we present the mathematical reasons why allocating the total project contingency to the individual risks is likely to result in significant cost overruns and/or project failure. We 
demonstrate these ideas using a simplified project with two risks. The formulation can readily be extended to multiple risks.

\section{A SIMPLE EXAMPLE}

\subsection{Summary of Risk Analysis}

We consider a project with two risks, $\mathrm{R} 1$ and $\mathrm{R} 2$, and the following assumptions:

(1) R1 and R2 are independent. The outcome of R1 does not affect the outcome of R2 and vice versa. This assumption is valid if the risks are associated with different and individually managed subsystems.

(2) There is a $30 \%$ probability that $\mathrm{R} 1$ will strike. Given that R1 strikes there is a distribution of outcomes with associated probabilities. The conditional outcomes are parameterized with a triangular distribution with the following parameters: minimum $=\$ 10 \mathrm{~K}$, most-likely value $=$ $\$ 30 \mathrm{~K}$, maximum $=\$ 70 \mathrm{~K}$. The possible outcomes and associated cumulative risk profile are shown in Figure 1.

(3) R2 is also given by Figure 1. This is simply done to avoid introducing new data.

We follow the standard decision tree convention (PrecisionTree ${ }, 1998$ ). Circles depict chance nodes; branches depict the possible outcomes. A branch with a continuous probability distribution represents a continuum of outcomes. The triangular distribution is specified as TRAND(minimum, most likely value, maximum). The cumulative risk profile explicitly marks a few values used in the subsequent discussion. These points are labeled as (cost-risk, probability that value is not exceeded).

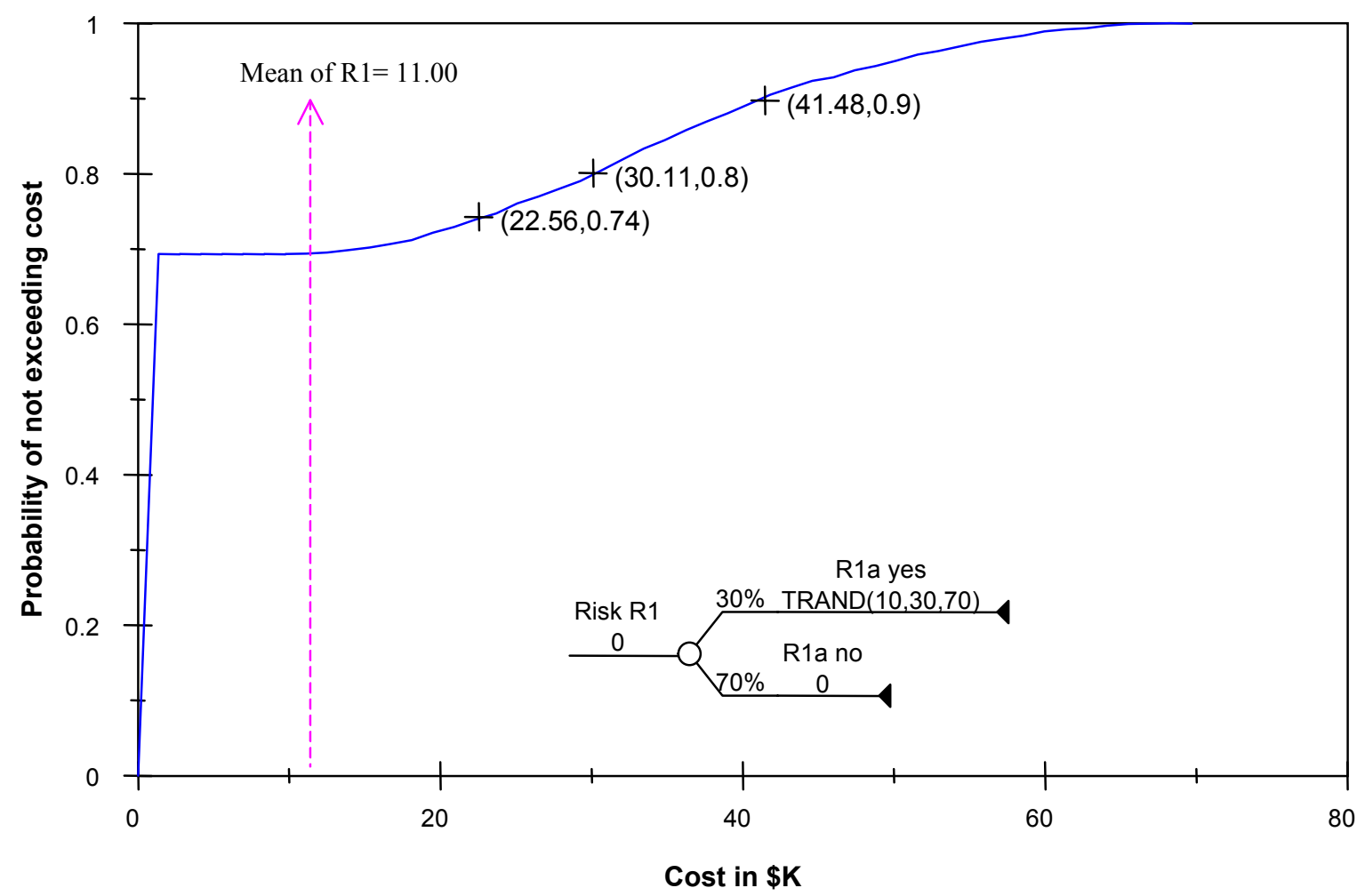

Figure 1. Risk R1 event tree and cumulative risk profile. 
The total project residual risk is the probabilistic sum of risks R1 and R2. Figure 2 depicts the associated event tree and cumulative risk profile. The reported results represent 5,000 Monte Carlo trials simulated using DecisionPro ${ }^{\circledR}$ [Vanguard Software, 1998]. Identical results can be obtained using commercially available Excel ${ }^{\circledR}$ add-ins such as $@$ Risk $®$ and Crystal Ball $®$.

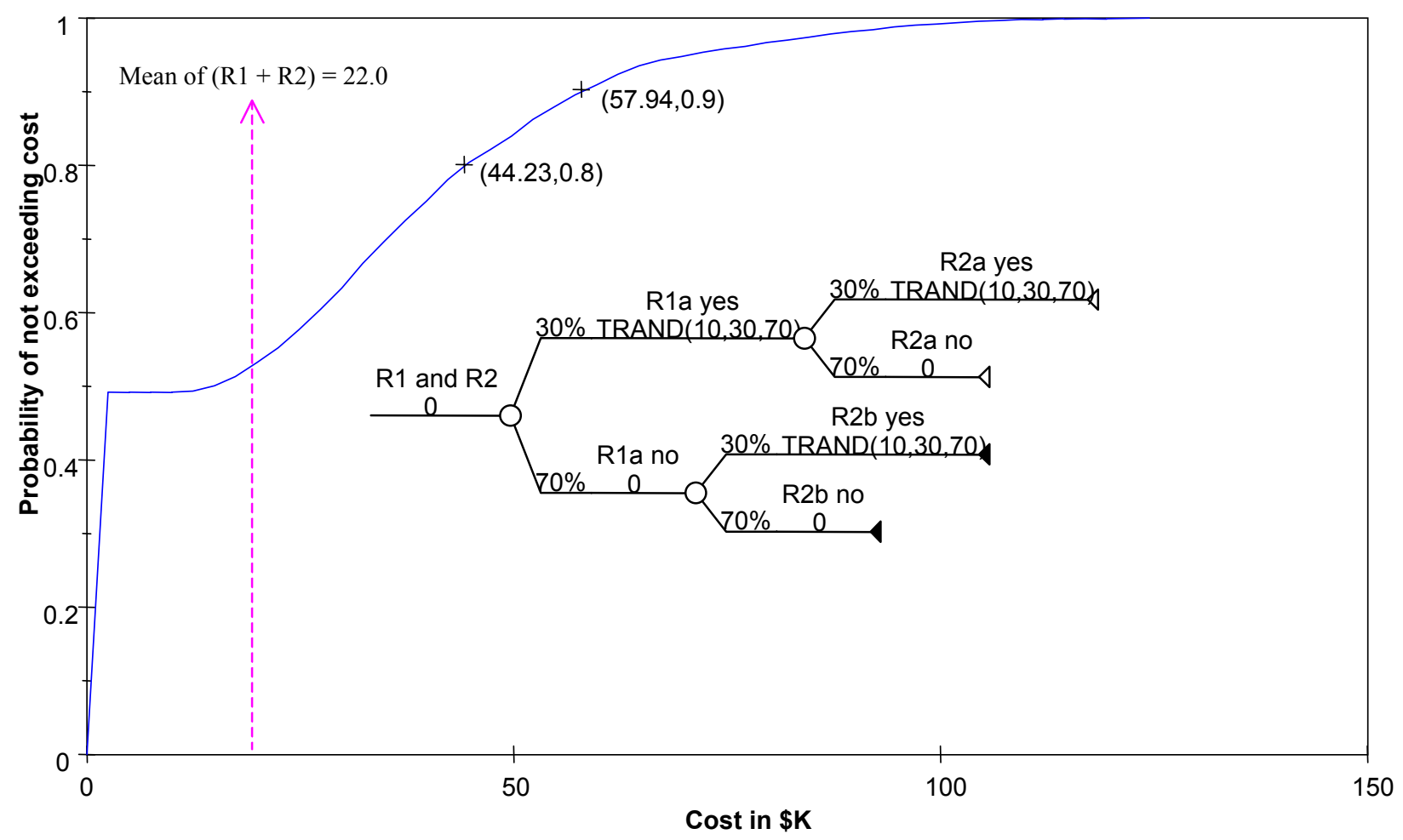

Figure 2. Total project risk event tree and cumulative risk profile.

\subsection{The Subsystem Managers Request Individual Contingencies}

Each subsystem manager analyses the data in Figure 1. Each agrees that the respective risk models and quantification are reasonable. Each wants the project to succeed and consequently thinks that the subsystem cost estimate should be at the $90 \%$ confidence level. Each subsystem manager requests a contingency of $\$ 42 \mathrm{~K}$.

\subsection{The Project Manager Acts}

The project manager finds the above analysis very interesting and to his liking. The subsystem managers responsible for risks R1 and R2 had each argued that they needed a cost contingency of $\$ 42 \mathrm{~K}$. The project manager justifiably thinks that the two managers are very capable but riskaverse. By analyzing Figure 2, he concludes that he can achieve an $80 \%$ probability of success with a cost contingency of $\$ 44 \mathrm{~K}$. Since the two risks are quantitatively identical, he allocates $\$ 22 \mathrm{~K}$ to each subsystem manager. The project manager is very pleased that he is able to cut the total project contingency almost in half and still maintain the contractually agreed-to probability of success. 


\subsection{The Outcome}

Both subsystem managers work very hard. Risk R1 does not strike and the responsible subsystem manager uses his budget to implement additional features. Risk R2 strikes hard and the responsible subsystem manager cannot mitigate the consequences with the $\$ 22 \mathrm{~K}$ he has been allocated. He estimates that he needs another $\$ 15 \mathrm{~K}$ to successfully develop his subsystem. The project manager is very surprised when he runs out of budget and the project is cancelled.

\subsection{The Risk Analyst Explains That The Analysis Was Misused}

The risk analyst is not surprised that the project cost exceeds the total cost estimate because he understands that the project manager has unwittingly reduced the effectiveness of the total project cost contingency and lowered the probability of success. Another smart individual had stumbled on a probability puzzler [Nahin, 2000]. The risk analyst explains the analysis and outcome as follows:

1. A project-wide cost contingency of $\$ 44 \mathrm{~K}$ is adequate for an $80 \%$ probability of success provided that it is available to either risk when needed. The mathematical analysis in Figure 2 assumes that the contingency usage is probabilistic on a project-wide level.

2. Contingency allocation represents a decision process and thus is not adequately modeled by the chance nodes of the event tree in Figure 2. The cumulative risk profile in Figure 2 is not valid for the situation where contingencies are allocated or committed to individual risks.

3. With a contingency of $\$ 22 \mathrm{~K}$, each subsystem manager only has a $74 \%$ probability of meeting his cost estimate (See Figure 1).

4. Given that each subsystem has a $74 \%$ probability of success, the project only has a $55 \%$ probability of success. This is significantly less than the $80 \%$ probability of success or confidence level that the project manager had assumed.

5. Algebraically summing the individual contingencies is not valid. The algebraic sum of the $80 \%$ confidence level cost risks equals $\$ 60 \mathrm{~K}$ (See Figure 1). It is significantly higher than the true $80 \%$ confidence level total project cost risk of $\$ 44 \mathrm{~K}$ (See Figure 2).

6. Only the mean values sum algebraically. But, it can be seen from Figures 1 and 2 that that risk is not adequately characterized by its mean value because decision-makers are generally more concerned with the magnitude of the downside distribution of outcomes than the probability. Empirical studies [Shapira, 1995: pp. 51] confirm that a majority of project managers agree with this statement and that they want valid information rather than simplicity when making high-risk decisions.

The risk allocation options can be graphically represented using the concept of reliability block diagrams as depicted in Figure 3. When the contingency is held at the project-wide level, the full amount is available to either risk and the situation is modeled in Figure 3a. When the contingency is allocated to individual risks, it is no longer available to the other risks on an asneeded basis. Furthermore when a risk strikes, the impact is likely to be severe and a small contingency is inadequate to provide success. In the present example, a contingency of $\$ 22 \mathrm{~K}$ only raises the probability of success of an individual subsystem from $70 \%$ to $74 \%$. The situation is modeled in Figure 3b. Using basic reliability theory [Shooman, 1968], it can readily be shown that the Figure 3a option is more reliable than the Figure $3 \mathrm{~b}$ option and that the results are consistent with the risk analyst's assessment. 


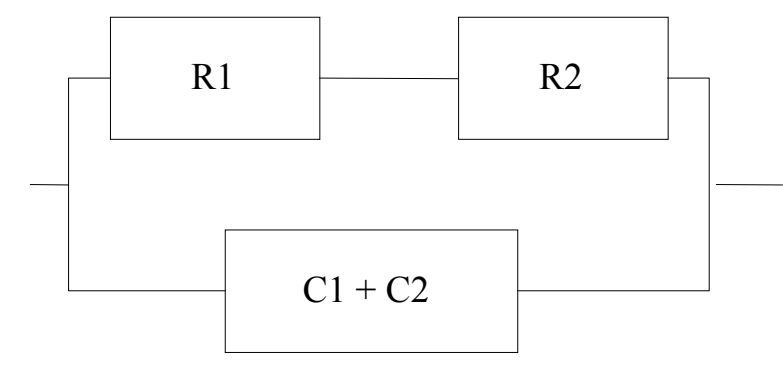

Total project succeeds if $(\mathrm{R} 1+\mathrm{R} 2)<=(\mathrm{C} 1+\mathrm{C} 2)$

Figure 3a. Contingencies $\mathrm{C} 1 \& \mathrm{C} 2$ are available to both R1 \& R2

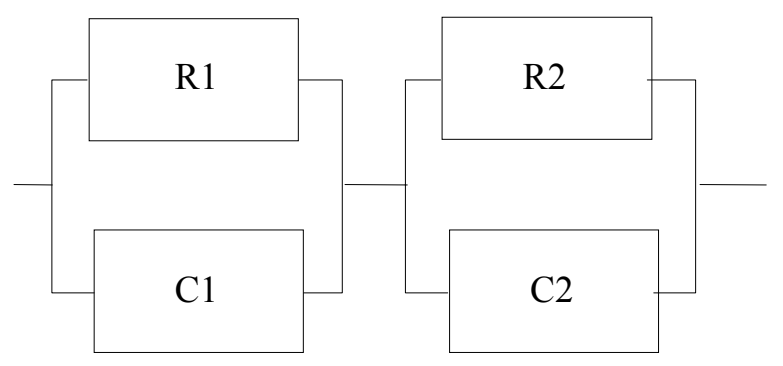

$\mathrm{R} 1$ succeeds if $\mathrm{R} 1<=\mathrm{C} 1$

$\mathrm{R} 2$ succeeds if $\mathrm{R} 2<=\mathrm{C} 2$

Total project succeeds if

both R1 succeeds and R2 succeeds

Figure 3b. Contingency $\mathrm{C} 1$ is available $\mathrm{R} 1$

Contingency $\mathrm{C} 2$ is available $\mathrm{R} 2$

Figure 3. Equivalent reliability block diagrams for modeling contingency allocation.

\section{CONCLUDING REMARKS}

The reported example illustrates the following important principles for efficiently managing cost contingencies:

1. Total project cost contingency versus probability of success is mathematically determined by probabilistically (not algebraically) summing the individual residual risks.

2. A project-wide cost contingency should be established to ensure an agreed-to probability of success or confidence level.

3. Contingencies should not be allocated at the task-level and held by individual subsystem managers. They should be held and managed at the project-wide level.

All three principles are necessary to ensure that adequate contingencies are available to mitigate all risks and not just selected ones. Projects that implement all three principles are more likely to succeed and to cost less. This requires an integrated approach to risk management and project management [Paté-Cornell and Dillon, 2001].

\section{ACKNOWLEDGMENT}

The author would like to thank Bill Edwards of the Lawrence Berkeley National Laboratory for insightful discussions and thoughtful comments on the manuscript. This work was supported by the Director, Office of Science, Office of Basic Energy Sciences, of the US Department of Energy under Contract No. DE-AC03-76SF00098. 


\section{REFERENCES}

E.M. Goldratt, Critical Chain, North River Press, Great Barrington, 1997.

J.P. Kindinger, Use of Probabilistic Cost and Schedule Analysis Results for Project Budgeting and Contingency Analysis at Los Alamos National Laboratory, Proceedings of the $30^{\text {th }}$ Annual Project Management Institute 1999 Seminars \& Symposium, http://www.risksig.com/articles/pmi2000/rk06.pdf.

H.M. Markowitz, Portfolio Selection: Efficient Diversification of Investments, Blackwell Publishers, Malden, 1991.

P.J. Nahin, Duelling Idiots and Other Probability Puzzlers, Princeton University Press, Princeton, 2000.

M.E. Paté-Cornell and R.L. Dillon, "Success Factors and Future Challenges in the Management of Faster-Better-Cheaper Projects: Lessons Learned from NASA," IEEE Transactions on Engineering Management, Vol. 48, No. 1 (February 2001), 25-35.

PrecisionTree ${ }^{\circledR}$, Palisade Corporation, http://www.palisade.com.

Z. Shapira, Risk Taking: A Managerial Perspective, Russell Sage Foundation, New York, 1995. M.L. Shooman, Probabilistic Reliability: An Engineering Approach, McGraw-Hill, New York, 1968.

U.S. Department of Energy, Project Management Practices, Draft, October 2000.

Vanguard Software Corporation, Decision Pro 3.0®, http://www.vanguardsw.com. 